\title{
Size effect in the electronic transport of thin films of $\mathrm{Bi}_{2} \mathrm{Se}_{3}$
}

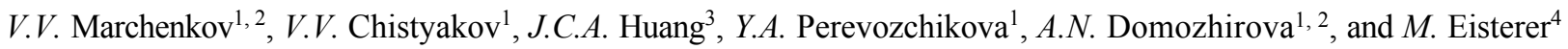

${ }^{1}$ M.N. Mikheev Institute of Metal Physics, UB RAS, 620137 Ekaterinburg, Russia

${ }^{2}$ Ural Federal University, 620002 Ekaterinburg, Russia

${ }^{3}$ National Cheng Kung University, Tainan, Taiwan

${ }^{4}$ TU Wien Atominstitut, 1020 Vienna, Austria

\begin{abstract}
Thin films of a topological insulator (TI) $\mathrm{Bi}_{2} \mathrm{Se}_{3}$ of various thicknesses from $20 \mathrm{~nm}$ to $75 \mathrm{~nm}$ were obtained. The resistivity measurements were carried out according to the conventional 4-contact DC technique. This allows to "separate" the bulk and surface conductivities at different temperatures and magnetic fields. It was suggested that similar effects should be observed in other TIs and systems with inhomogeneous distribution of dc-current on sample cross section.
\end{abstract}

\section{Introduction}

The new functional materials with the unique physical properties are needed for spintronic devices. One of such promising materials are the topological insulators (TIs) [1], which have a nontrivial topological band structure, arising from strong spin-orbital interaction [2]. The TI has an energy gap and, hence, is the insulator or semiconductor, in a bulk and has the protected gapless conduction states on its surface. A rigid connection between the directions of the momentum and the electron spin leads to the emergence of spin polarization of charge carriers and the possibility of a spin-polarized current flowing near the TI surface with practically no loss [3]. This spin-polarized surface current can be used for spintronic devices.

It is known that $\mathrm{Bi}_{2} \mathrm{Se}_{3}$ compound is the TI $[4,5]$ with a metallic conductivity near surface and the gapless semiconductive one [6] in its bulk. Since the electroconductivity value in a bulk and near surface of such materials can differ substantially, it is of interest to "divide" them experimentally. For this, we can use the results of Ref. [7], where we studied the size effect in the conductivity of pure tungsten single crystals under conditions of the static skin effect (SSE) [8], i.e. a predominant flow of direct electric current near a sample surface. The aim of this paper is to search for and study the size effect in the electronic transport of thin films of $\mathrm{TI} \mathrm{Bi}_{2} \mathrm{Se}_{3}$.

\section{Samples and measurements}

Thin films of $\mathrm{Bi}_{2} \mathrm{Se}_{3}$ were grown by the molecular beam epitaxy method on $\mathrm{Al}_{2} \mathrm{O}_{3}$ substrates $[4,5]$ with thickness from 20 to $75 \mathrm{~nm}$. The XRD data and the atomic content of elements analysis showed the synthesized films have $\mathrm{Bi}_{2} \mathrm{Se}_{3}$ composition (see Refs. [4, 5]). The atomic content of elements was measured by a scanning electron microscope equipped with an EDAX X-ray microanalysis attachment. Our examination showed that the deviations from a stoichiometric composition were insignificant in all samples. The measurements of the electroresistivity $\rho_{0}$ and magnetoresistivity $\rho_{x x}$ were carried out by the conventional 4-points method at dccurrent in the temperature range from 4.2 to $80 \mathrm{~K}$ and in magnetic fields of up to $10 \mathrm{~T}$. The results are presented in units of conductivities $\sigma_{0} \approx 1 / \rho_{0}$ and $\sigma_{x x} \approx 1 / \rho_{x x}$.

\section{Results and discussion}

Schematic view of the thin film of TI is shown in Fig.1. The electric current $I$ is passed through the film of thickness $d$ and width $c$ (Fig. 1), and the voltage $U$ is measured between the potential leads located at a distance $L$. In the near-surface layer of thickness $\delta$, a region with high surface conductivity $\sigma^{\text {sur }}$ appears.

Total conductivity of such a system contains two terms, namely, the surface conductivity $\sigma^{\text {sur }}$ and the bulk conductivity $\sigma^{\text {bulk }}$. It is quite similar to Refs. $[7,8]$, where the dc-current is concentrated near a sample surface due to the static skin effect (SSE) [8]. It is quite easy to show that

\footnotetext{
* Corresponding author: wchist@imp.uran.ru
} 


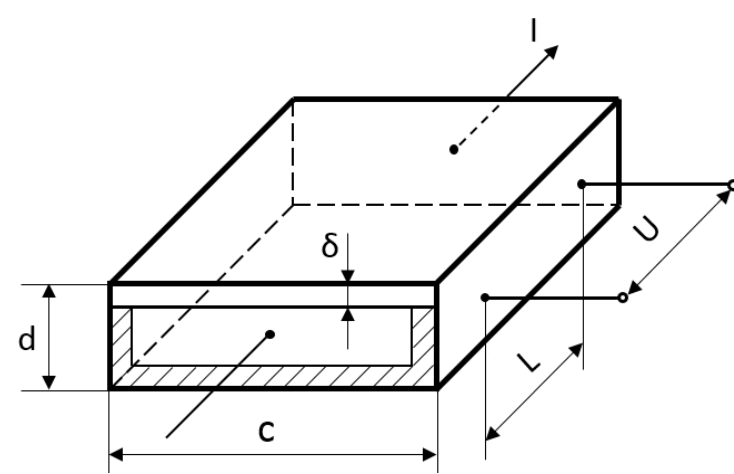

Fig. 1. The schematic view of experiment. Where $c$ is the width of the sample; $d$ is the thickness of the sample; $\delta$ is the thickness of the near-surface layer; $L$ is the distance between potential contacts, $I$ is dc current, $U$ is measured voltage.

$$
\sigma \approx \sigma^{s u r} \cdot(\delta / d)+\sigma^{b u l k}
$$

Thus, a linear dependence of the conductivity on the film thickness should be observed

$$
\sigma=f\left(d^{-1}\right)
$$

The first term in Eq.(1) is proportional to surface conductivity $\sigma^{\text {sur }}$ and second one is the bulk conductivity $\sigma^{b u l k}$, i.e. we can "separate" $\sigma^{\text {sur }}$ and $\sigma^{\text {bulk }}$. To do this the films of different thickness $d$ were synthesized. Fig. 2 presents the experimental results for conductivity of $\mathrm{Bi}_{2} \mathrm{Se}_{3}$ films without magnetic field (Fig. 2a) and in a field of $10 \mathrm{~T}$ at $\mathrm{T}=4.2 \mathrm{~K}$ (Fig. $2 \mathrm{~b}$ ). One can see that there is a linear dependence on $d^{-1}$ both for $\sigma_{0}$ and $\sigma_{x x}$. It allowed us to separate the bulk and surface conductivities. Taking into account Refs [11], it was assumed that delta is not more than $1 \mathrm{~nm}^{*}$.

According to estimations (evaluations) at $\mathrm{T}=4.2 \mathrm{~K}$, $\sigma_{0}{ }^{\text {bulk }} \sim 0.5 \cdot 10^{3}$ and $\sigma_{0}{ }^{\text {sur }} \sim 9.8 \cdot 10^{4}$ without magnetic field and $\sigma_{x x}{ }^{\text {bulk }} \sim 0.2 \cdot 10^{3}$ and $\sigma_{x x}$ sur $\sim 4.8 \cdot 10^{4}$ in a field of $10 \mathrm{~T}$. That means the surface conductivity $\sigma^{\text {sur }}$ is almost 200 times higher than $\sigma^{b u l k}, \sigma_{0}{ }^{\text {sur }}, \sigma_{x x}^{\text {sur }}>>\sigma_{0}^{\text {bulk }}, \sigma_{x x}^{\text {bulk. The }}$ obtained results are in good qualitative agreement with the Ref. [6].

Fig. 3a and Fig. 4a show the temperature dependence of $\sigma_{0}{ }^{\text {sur }}$ and $\sigma_{0}{ }^{\text {bulk }}$ without field. $\sigma_{0}{ }^{\text {sur }}$ decreases with temperature as it should be for protected conductive states on TI surface. $\sigma_{0}^{\text {bulk }}$ also decreases with $\mathrm{T}$ as it was observed for $\mathrm{Bi}_{2} \mathrm{Se}_{3}$ in Ref. [6] since $\mathrm{Bi}_{2} \mathrm{Se}_{3}$ has relatively small gap in its electron energy spectrum at Fermi level. It leads to that fact the scattering of charge carriers begin to play the main role in formation of its conductivity. As a result, $\sigma_{0}{ }^{\text {bulk }}$ decreases with temperature due to the scattering processes.

Magnetic field should change a type of bulk conductivity, as it should be in semiconductors with small gaps and in gapless semiconductors. Fig. 3b, 4b present the temperature dependence of the magnetoconductivities $\sigma_{x x}^{\text {sur }}$ and $\sigma_{x x}$ bulk. It is seen that $\sigma_{x x}{ }^{\text {sur }}$ decreases with temperature, and it is typical for systems with metallic states. $\sigma_{x x}$ bulk increases with temperature, which can be explained by changing the character of current carriers scattering for systems in which the scattering processes play the main role in conductivity.

It should be noted that a similar change in a type of temperature dependence for surface and bulk conductivities was observed in Refs. [9, 10], where dc- current was concentrated near a samples surface under the SSE [7, 8].

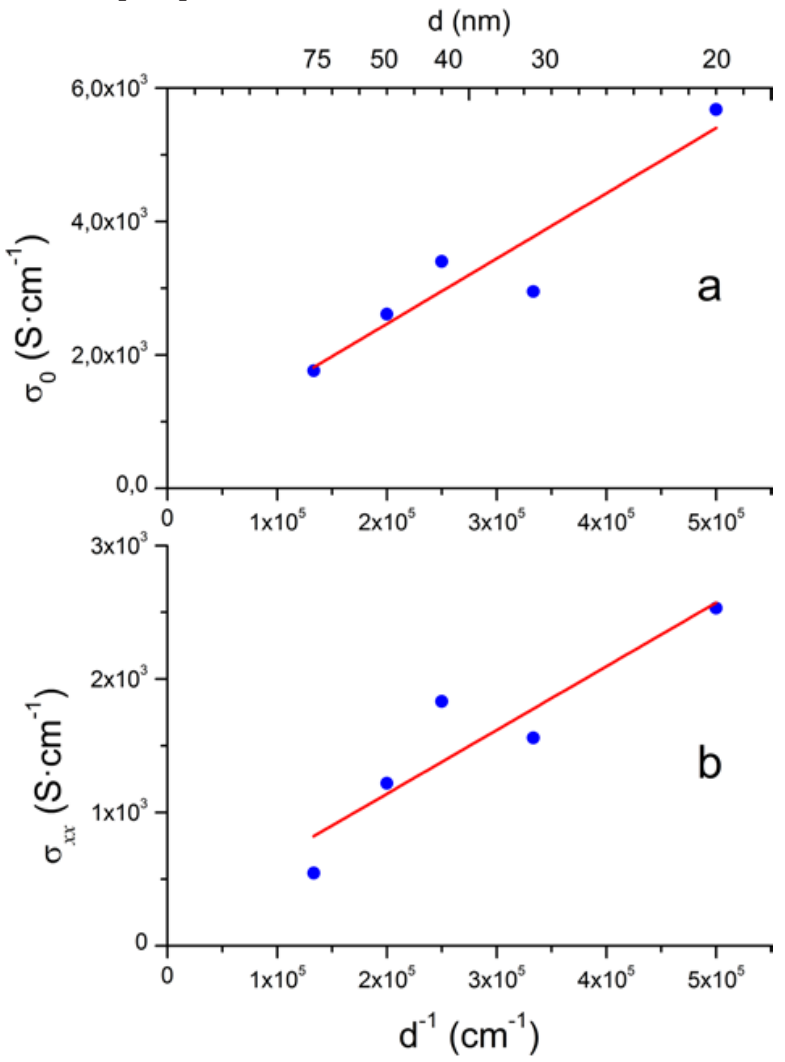

Fig.2. Size effect in the conductivity of thin films of $\mathrm{Bi}_{2} \mathrm{Se}_{3}$ in magnetic fields $0 \mathrm{~T}$ (a) and $10 \mathrm{~T}$ (b) at $4.2 \mathrm{~K}$.

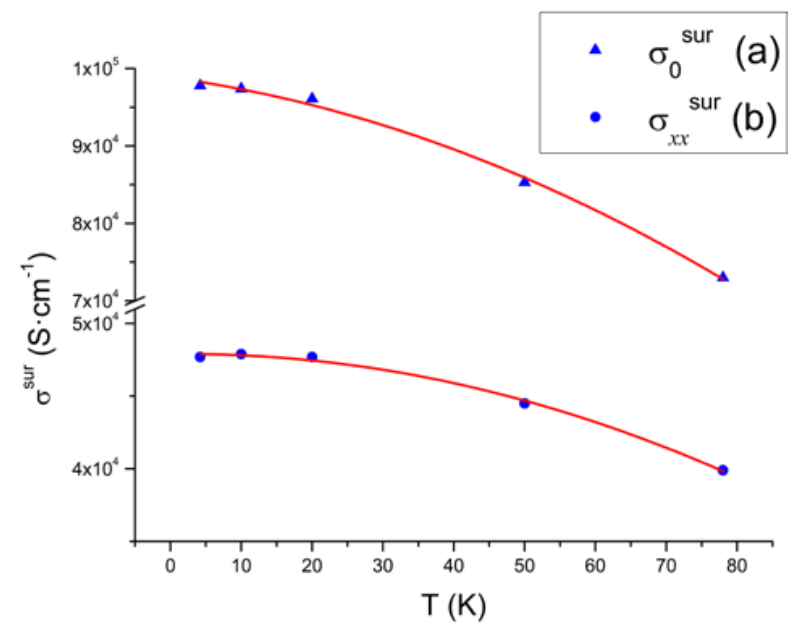

Fig. 3. Temperature dependences of surface conductivity of thin films $\mathrm{Bi}_{2} \mathrm{Se}_{3}$ in magnetic fields 0T (a) and $10 \mathrm{~T}$ (b).

*It is an estimation from "above", which gives a smaller value of $\sigma^{\text {sur }}$. 


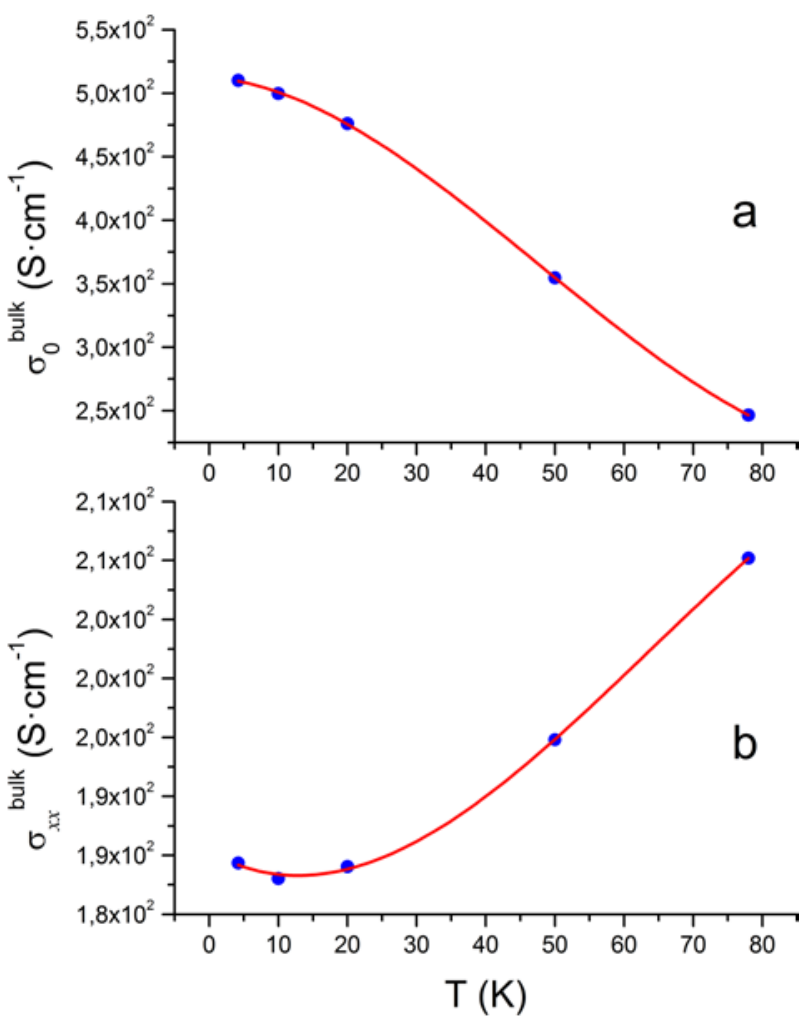

Fig. 4. Temperature dependences of bulk conductivity of thin films $\mathrm{Bi}_{2} \mathrm{Se}_{3}$ in magnetic fields 0T (a) and 10T (b).

\section{Conclusions}

The size effect, i.e. a dependence of the conductivity on the film inverse thickness, was observed in thin films of TI $\mathrm{Bi}_{2} \mathrm{Se}_{3}$. This allows us to "separate" the bulk and surface conductivities at different temperatures and magnetic fields. In apparently, similar effects should be observed in other TIs and systems with inhomogeneous distribution of dc-current on sample cross section.

This work was partly supported by the state assignment of FASO of Russia (theme "Spin" No. AAAAA18-118020290104-2), by the RFBR (project No. 17-52-52008), by the Government of the Russian Federation (state contract No. 02.A03.21.0006), and by grant of Russian Ministry of Education and Science No 14.Z50.31.0025.

\section{References}

1. H. Zhang, C.X. Liu et al., Nat. Phys. 5, 438 (2009)

2. S.Y. Xu, I. Belopolski, et al., Science 349, 6248 (2015)

3. Z.K. Liu, L.X. Yang et al., Nat. Mat. 15, 27 (2016)

4. Y.H. Liu, C.W. Chong et al., Appl. Phys. Lett. 107, 012106 (2015)

5. Y. Liu, C. Chong, W. Chen et al., Jpn. J. Appl. Phys. 56, 070311 (2017)

6. B. Bhattacharyya, A. Sharma et al., J. Phys.: Condens. Matter 29, 115602 (2017)
7. V.V. Marchenkov, H.W. Weber, J. Low Temp. Phys. 132, 135 (2003)

8. N.V. Volkenshtein, M. Glinski et al., Sov. Phys. JETP 68, 1216 (1989)

9. V.V. Marchenkov, A.N. Cherepanov et al., J. Low Temp. Phys. 98, 425 (1995)

10. V.V. Marchenkov, H.W. Weber et al., J. Low Temp. Phys. 102, 133 (1996)

11. X.L. Qi, S.C. Zhang, Rev. Mod. Phys. 83, 1057 (2011) 